\title{
Modifying the Environment and Policy Assessment and Observation (EPAO) to better capture feeding practices of family childcare home providers
}

\author{
Alison Tovar ${ }^{1, *}$, Amber E Vaughn ${ }^{2}$, Jennifer Orlet Fisher ${ }^{3}$, Sara E Benjamin Neelon ${ }^{4}$, \\ Regan Burney ${ }^{5}$, Kathleen Webster ${ }^{6}$, Tao Liu ${ }^{7}$, Truls Ostbye ${ }^{8}$ and Dianne S Ward ${ }^{9}$ \\ 'Department of Nutrition and Food Sciences, University of Rhode Island, Kingston, RI 02881, USA: ${ }^{2}$ Children's \\ Healthy Weight Research Group, UNC Center for Health Promotion \& Disease Prevention, University of North \\ Carolina at Chapel Hill, Chapel Hill, NC, USA: ${ }^{3}$ Department of Social and Behavioral Sciences, Center for Obesity \\ Research and Education, College of Public Health, Temple University, Philadelphia, PA, USA: ${ }^{4}$ Johns Hopkins \\ Bloomberg School of Public Health, Baltimore, MD, USA: ${ }^{5}$ Center for Health Promotion and Disease Prevention, \\ University of North Carolina at Chapel Hill, Chapel Hill, NC, USA: ${ }^{6}$ Department of Psychology, University of Rhode \\ Island, Kingston, RI, USA: ${ }^{7}$ Department of Biostatistics, Center for Statistical Sciences, Brown University, Providence, \\ RI, USA: ${ }^{8}$ Community and Family Medicine, Nursing and Global Health, Duke University Medical Center, Durham, \\ NC, USA: ${ }^{9}$ Department of Nutrition, UNC Center for Health Promotion \& Disease Prevention, University of North \\ Carolina at Chapel Hill, Chapel Hill, NC, USA
}

Submitted 5 February 2018: Final revision received 31 August 2018: Accepted 7 September 2018: First published online 31 0ctober 2018

\begin{abstract}
Objective: To describe the modification and validation of an existing instrument, the Environment and Policy Assessment and Observation (EPAO), to better capture provider feeding practices.

Design: Modifications to the EPAO were made, validity assessed through expert review, pilot tested and then used to collect follow-up data during a two-day home visit from an ongoing cluster-randomized trial. Exploratory factor analysis investigated the underlying factor structure of the feeding practices. To test predictive validity of the factors, multilevel mixed models examined associations between factors and child's diet quality as captured by the Healthy Eating Index-2010 (HEI-2010) score (measured via the Dietary Observation in Childcare Protocol).

Setting: Family childcare homes (FCCH) in Rhode Island and North Carolina, USA. Participants: The modified EPAO was pilot tested with fifty-three FCCH and then used to collect data in $133 \mathrm{FCCH}$.

Results: The final three-factor solution ('coercive control and indulgent feeding practices', 'autonomy support practices', 'negative role modelling') captured $43 \%$ of total variance. In multilevel mixed models adjusted for covariates, 'autonomy support practices' was positively associated with children's diet quality. A 1-unit increase in the use of 'autonomy support practices' was associated with a 9.4-unit increase in child HEI-2010 score $(P=0 \cdot 001)$.

Conclusions: Similar to the parenting literature, constructs which describe coercive controlling practices and those which describe autonomy-supportive practices emerged. Given that diets of pre-schoolers in the USA remain suboptimal, teaching childcare providers about supportive feeding practices may help improve children's diet quality.
\end{abstract}

Keywords Childcare provider Feeding practices Observational tool
Early childhood is a critical period when dietary intake patterns and eating habits are developed ${ }^{(1-3)}$. Adult caregivers play an important role in children's socialization and in their development of behaviours, habits and attitudes, including those around food and eating ${ }^{(4,5)}$. Most research, however, has focused on parents, specifically their food parenting practices. Food parenting practices are parental behaviours (intentional or unintentional) that influence children's attitudes, behaviours or beliefs around food and eating ${ }^{(6)}$. This literature generally suggests that coercive practices (e.g. restriction, pressure to eat, food bribes) are associated with poorer dietary quality and eating habits, while autonomy-supporting and structure practices (e.g. encouragement, praise, nutrition education, modelling, 
food availability) are associated with better diet quality and eating habits ${ }^{(6)}$.

In the USA, about $30 \%$ of children under the age of 5 years are enrolled in some type of formal childcare programme, consuming up to two-thirds of their daily nutrients in this setting ${ }^{(7-11)}$. As a result, childcare providers have assumed much of the responsibility for child feeding ${ }^{(12,13)}$, but less is known about how their feeding practices may be shaping children's eating habits. Studies with childcare providers suggest that they use a variety of practices, including coercive, autonomy-supporting and structure practices $^{(14-18)}$. Provider feeding practices shown to promote healthier eating habits in children include sitting with children during meals ${ }^{(19)}$, being enthusiastic role models $^{(20-22)}$, involving children in meal preparation ${ }^{(23)}$ and talking with children about healthy foods ${ }^{(24,25)}$. While the child feeding parenting literature has been used to inform the thinking around how providers' practices might influence children's eating habits, childcare providers may not use the same practices as parents and these practices may have a different impact on children when used in the childcare setting $^{(26)}$.

To better understand how providers' feeding practices influence children's dietary intakes and eating behaviours, researchers must be able to measure these practices ${ }^{(27)}$. Unfortunately, tools assessing provider feeding practices are limited and have often relied on slightly modified parental feeding tools ${ }^{(28-30)}$. In addition, most studies conducted in childcare settings that have examined provider feeding practices have relied on single items, rather than constructs or scales, to measure these practices and assess their associations with the children's diet ${ }^{(23,29)}$. Furthermore, while these studies advanced the assessment of provider feeding practices, they have not used a consistent theoretical framework as a guide, limiting the ability to make comparisons and conclusions across studies ${ }^{(6)}$. Key characteristics of the childcare setting must be taken into consideration when trying to measure these practices or adapt existing parenting practices measures for use with providers. For example, providers' use of feeding practices is likely influenced by the fact that they are responsible for feeding multiple children at once, and they likely also feel pressure from parents to make sure that children in their care eat a sufficient amount of food. Because of this measurement gap, the goal of the present paper was to describe the modification and validation of an existing instrument, the Environment and Policy Assessment and Observation $(\mathrm{EPAO})^{(31)}$, to better capture provider feeding practices. Specifically, we describe the identification of missing feeding practice constructs, development of new items and results from the psychometric testing of new scales.

\section{Methods}

The EPAO is designed to assess nutrition and physical activity environments of childcare centres using a combination of direct observation of a full day of childcare and review of formal documents ${ }^{(32)}$. A version of this instrument was used as part of a larger cluster-randomized trial (Keys) evaluating the efficacy of an intervention designed to help family childcare home $(\mathrm{FCCH})$ providers become healthy role models, provide environments to support healthy eating and physical activity in children, and implement more effective business practices $^{(33)}$. An FCCH is a specific type of childcare setting that is relatively small (serving on average five children) which operates out of the provider's own residence. Approximately 3 million children, from birth to age 5 years, are cared for by these providers ${ }^{(8)}$. As part of this larger study, a version of the Environment and Policy Assessment and Observation modified for the family home setting $(\mathrm{EPAO}-\mathrm{FCCH})^{(34)}$ was developed and used to collect information pre- and post-intervention. The supplemental sub-study (described here) further modified this EPAOFCCH to expand its assessment of provider feeding practices by meal time and assess the psychometric properties of these new scales.

\section{EPAO modification}

To enhance the measurement of provider feeding practices, modifications were made to the observation component of the EPAO. Specifically, additions were made to three sections: morning meal, lunch and afternoon snack. During the tailoring of the EPAO for FCCH, these sections had already been expanded to incorporate additional items assessing provider feeding practices such as role modelling, rewards, praise and encouragement ${ }^{(34)}$ based on updated best practice recommendations for childcare ${ }^{(35,36)}$. Reliability and validity of these food practice scales have also been demonstrated in studies with $\mathrm{FCCH}^{(34)}$ and Head Start centres $^{(37)}$.

While these studies advanced the assessment of provider feeding practices, comparison against a comprehensive content map of parental feeding practices ${ }^{(6)}$ suggested that there may be several constructs applicable to providers that were still not being assessed. This content map organizes feeding practices into three higher-order constructs: Coercive Control, Structure and Autonomy Support. Within each of these higher-order constructs, specific feeding practices are identified. 'Coercive control practices' reflect attempts to dominate, pressure or impose the will of the parents upon the child. 'Structure-related practices' reflect the consistent enforcement of rules and boundaries about eating, and the physical organization of the food environment. 'Autonomy support practices' provide sufficient structure within which the child can be involved in making food choices at a developmentally appropriate level, engaging in conversations with the child about reasons for rules and boundaries regarding food, and creating an emotional climate during these parent-child food interactions in which the child feels unconditionally loved, valued and accepted by parents. Using the content map, we were able 


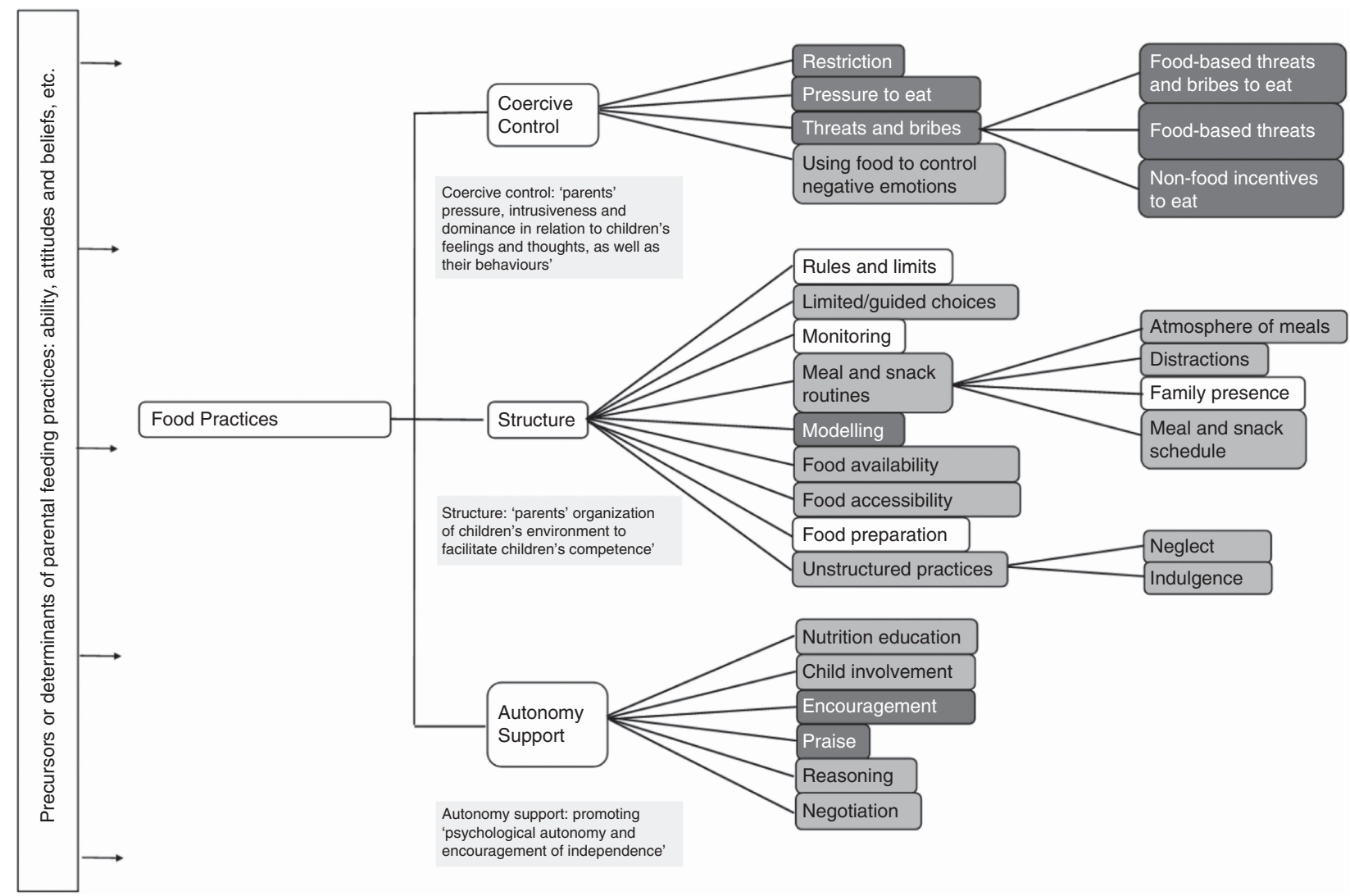

Fig. 1 Existing content map of feeding with existing and additional EPAO items: $\square$, measured by EPAO; $\square$, additions made by EPAO Supplemental Assessment of Feeding Practices (EPAO, Environment and Policy Assessment and Observation)

to identify practices that were already well measured in the EPAO, those that were partially measured, and those that were not measured but applicable to childcare providers (Fig. 1).

While this content map provided a useful guide for identifying potential feeding practice constructs, we were cognizant that not all parental feeding practices would be applicable to childcare providers. To help inform our selection of the most relevant feeding practice constructs, we conducted observations in forty-eight FCCH, observing 200 meals (breakfast, lunch and snack) consumed by children $^{(38)}$. These observations helped identify the feeding practices commonly used by providers as they interacted with children during meals (e.g. use of encouragement, reasoning and role modelling, as well as insistence, pressure and threats in response to children's food refusals). At this time a literature review was also conducted to identify existing tools attempting to measure provider feeding practices and relevant items extracted ${ }^{(15,39)}$. In addition to the existing items ( $n$ 23), twenty-five new provider feeding practice items were identified, reviewed by research team members and added to the EPAO. Response options for all feeding items prompted data collectors to note whether the practices were not observed, observed one or two times, or observed three or more times. For each of the new items, a brief behavioural definition was also developed to facilitate data collection accuracy during observations (available upon request from the corresponding author). Face and content validity were assessed by three content area experts. These experts reviewed draft items and provided feedback on their content, relevance and potential modifications.

\section{Pilot testing new items}

To evaluate the feasibility of data collection with these new items, two pilot studies were conducted sequentially. The first pilot study involved video-recording and subsequent coding of feeding sessions in five FCCH in Rhode Island. Protocols for this pilot were approved by the Institutional Review Boards at the University of Rhode Island. FCCH providers were identified based on the list provided from the Department of Children, Youth and Families website. A recruitment visit was used to review the data collection protocol, collect provider consent, distribute parent consent letters and schedule data collection (capturing up to four meal occasions, with at least two lunches and one snack). Data collection from each FCCH was completed within three days. On the first day of data collection in each FCCH, a video recorder (Sony camcorder) was placed on a tripod stand near the table where children were eating. Set-up occurred prior to the first observed meal occasion so that providers could be 
shown how to use the camcorder (e.g. how to turn it on/off at the beginning and end of meals). FCCH providers were then responsible for recording all subsequent meals. Videos were then coded by A.T. (lead author) and a research assistant using the new EPAO items. These data were used to calculate the frequency of each practice across all meals and snacks for each FCCH. Video clips were also extracted from these recordings to help create a 90-min training for data collectors that could provide clear examples for all practices captured in the new EPAO items.

A second pilot study was then conducted to assess the feasibility of using the tool for real-time coding with a sample of seventeen FCCH in North Carolina, using a convenience sample of FCCH already participating in the Keys study. Protocols for this second pilot were approved by the Institutional Review Boards at the University of North Carolina at Chapel Hill, Duke University and the University of Rhode Island. EPAO data, including these new items, were collected in real time during two-day onsite visits. Prior to data collection, all data collectors were trained on the modified EPAO using the training video described above. Following data collection, an indepth feedback session was conducted with the data collectors to inquire about any confusion about items or questions about how items should be operationalized. For six of the homes, two data collectors completed the EPAOFCCH with new feeding practice items, thus allowing assessment of reliability. Results found that $80 \%$ of the new items had substantial to high inter-rater agreement $(\kappa=0 \cdot 60-0 \cdot 99)$.

\section{Psychometric testing of provider food practice items}

The EPAO Supplemental Assessment of Feeding Practices was incorporated into the follow-up data collection in the ongoing Keys study ${ }^{(33)}$. Given the timing of grant funds for this project, the final version of the tool was available for follow-up data collection with only 133 of the 166 FCCH participating in Keys. Per Keys protocols, a two-day onsite visit was conducted with each FCCH. The EPAO-FCCH and the Supplemental Assessment of Feeding Practices were completed on both days. Child dietary intake while at the FCCH (not what is consumed in their home) was also assessed on these days using the Diet Observation in Childcare (DOCC) ${ }^{(40)}$. According to DOCC protocol, one observer can accurately observe and record a maximum of three children. Typically, this included breakfast/morning snack, lunch and an afternoon snack. Data collectors estimated the quantity of foods and beverages served, added (i.e. second helpings), exchanged, wasted and remaining following the end of each meal and snack to calculate the total quantity served to and consumed by children. If additional detail was needed about foods or beverages served (e.g. preparation of mixed dishes), the data collector would request this information from the FCCH provider. Valid diet observation days had to capture lunch and at least one additional meal or snack, thus setting a minimum level of data required for each day given that the number of meals and snacks served can vary by FCCH and child (depending on the hours they are enrolled in care). If a child was absent or left early (before sufficient dietary data could be collected), an additional visit was conducted to repeat the diet observation for that child. DOCC data were analysed using the Nutrition Data System for Research (NDSR) software (Nutrition Coordinating Center, University of Minnesota, Minneapolis, MN, USA; http://www.ncc.umn.edu/products/) and used to calculate a daily Healthy Eating Index-2010 (HEI-2010) score ${ }^{(41)}$ for each child. As per protocol, diet data for each child were first summed across the two observation days and then HEI-2010 score was calculated from this sum. HEI-2010 was designed to measure diet quality in terms of how well diets conform to the 2010 Dietary Guidelines for Americans ${ }^{(42)}$. The total HEI-2010 score represents the sum of twelve component scores (maximum component score shown in parentheses), including: total fruit (5), whole fruit (5), total vegetables (5), green and beans (includes dark green vegetables and cooked, dried beans and peas because intakes of these types of vegetables are furthest from the amounts recommended in the US Department of Agriculture Food Patterns) (5), whole grains (10), dairy (10), total protein food (5), seafood and plant proteins (5), fatty acids (10), refined grains (10), sodium (10) and empty calories (20). Total HEI-2010 scores can have a maximum value of 100 which indicates high diet quality ${ }^{(43)}$. Prior studies have utilized the HEI-2010 to evaluate overall diets as well as specific meals for pre-schoolers and have shown that it is a valid method in this context ${ }^{(44-46)}$. The index adjusts per $4184 \mathrm{~kJ}$ ( $1000 \mathrm{kcal})$; hence it is not necessary to further adjust for the number of meals.

\section{Data analysis}

Data on provider feeding practices, which had been collected over two days and multiple meals and snacks each day, were summarized into weighted average scores. For each meal or snack, the occurrence of each food practice was originally coded as $0,1-2$ or $3+$ times. For purposes of analysis, items were recoded as occurring $0,1.5$ or 3 times. To account for the different number of meals and snacks offered during the day and to account for longer meals (i.e. lunchtime), we then created a weighting factor for each 'meal and snack occasion', i.e. the average duration (minutes) of a meal/snack divided by the total duration for all meals and snacks that day. This weighting factor was then multiplied by the number of occurrences recorded during that meal/snack for a given food practice. Finally, these weighted occurrences at each meal and snack were summed over the entire day. For example, if there was a total of $43 \mathrm{~min}$ of meals and snacks, the weighted daily score for one food 
practice such as 'praised for eating new food' would be calculated as follows:

$$
\begin{aligned}
& \text { Morning meal }(10 \mathrm{~min} / 43 \mathrm{~min}) \times 1 \cdot 5 \text { (times) } \\
& +\operatorname{lunch}(25 \mathrm{~min} / 43 \mathrm{~min}) \times 3(\text { times }) \\
& +\operatorname{afternoon} \operatorname{snack}(8 \mathrm{~min} / 43 \mathrm{~min}) \times 1(\text { time })=2 \cdot 28
\end{aligned}
$$

This weighted daily score was calculated for each feeding practice each day, then averaged across the two observation days to obtain the weighted average score. These scores were calculated for all forty-eight feeding practices items, including the twenty-three pre-existing items and twenty-five newly developed items.

To inform an exploratory factor analysis (EFA), descriptive statistics and bivariate correlations were run on all forty-eight items. Regardless of significance levels, meaningful correlations were considered at an absolute value of $r \geq 0.40$. Consideration of redundancy of variance or the presence of multicollinearity was considered for bivariate correlations with $r>0 \cdot 80$.

The EFA was then run with all forty-eight items included in the analysis. To allow for correlation across items and resulting factors, extraction was set for oblimin (oblique) rotation over an orthogonal structure. We also examined the Kaiser-Meyer-Olkin index, a test for sampling adequacy with values ranging from 0 to 1 . The resulting index was 0.84 , well above the 0.6 recommendation for sufficient interrelationship between variables required for an EFA. Bartlett's test of sphericity was also significant with $\chi_{(1128)}^{2}=4324, P<0 \cdot 001$. Given that these constructs had not been tested before with childcare providers, no $a$ priori number of factors was selected for the initial EFA. To determine the number of underlying factors inherent in the data, the initial EFA was examined using scree plots, eigenvalues greater than 1 and percentage of variance captured for each resulting factor. To determine which items loaded on specific factors a 'simple structure' approach was used, where the presence of a compound loading was determined if there was less than a $\pm 0 \cdot 20 \beta$ value spread across all factors. When compound loadings existed, those items were removed from the analysis. Marker loadings with absolute values $\geq 0.40$ were considered meaningful and the item was retained on its respective factor. To create the final factors, we averaged the weighted average scores from all items within that factor (sum of all item scores within each factor divided by the number of items).

To test the predictive validity of the constructs, multilevel mixed-effects models were used to examine the association between the three provider feeding practices (independent variable) and child's diet quality as captured by the HEI-2010 score (dependent variable). The three food practice variables were standardized to have a mean of 0 and an SD of 1 , so their regression coefficients captured the effect on HEI-2010 score for a 1 SD difference in the practices. The models were estimated using the method of generalized estimating equations ${ }^{(47)}$ and models included a random intercept to account for nesting of children within FCCH. In the initial model, we included all provider feeding practices and an a priori selected set of covariates, specifically provider income, education, age, race, Child Adult Care Food Program (CACFP) participation, childcare quality rating and BMI, as well as child age, sex, BMI, hours spent in childcare and study arm (intervention $v$. control). Feeding practices and covariates that did not contribute significantly to the model $(P<0 \cdot 10)$ were removed in a final reduced model. After excluding missing values for the dependent and independent variables, the final model included 125 of the $133 \mathrm{FCCH}$ (94\%).

Calculation of weighted average scores, descriptive analysis of feeding practice scores and reliability testing were completed in the statistical software package SAS version 9.4. The exploratory factor analysis was completed in IBM SPSS Statistics version 24. Predictive validity analysis was completed in $\mathrm{R}$ version 3.4.0.

\section{Results}

Of the $133 \mathrm{FCCH}$ providers participating in the present study, all were female, mean age was $49 \cdot 8$ (SD 9.4) years, $71.8 \%$ were African American, $72.1 \%$ had an associate or college degree, and $91.6 \%$ of their $\mathrm{FCCH}$ participated in CACFP. Of the participating children, $49.3 \%$ were female and their mean age was 3.3 (SD 1.2 ) years. This is similar to FCCH providers nationally in that most are female and most participate in $\mathrm{CACFP}^{(48)}$.

\section{Frequency of feeding practices}

When looking at simple presence $v$. absence, the positive feeding practices most frequently observed (seen in $90 \%$ or more of homes) included: encouraging children to try the foods on their plates $(96 \cdot 2 \%)$, talking with children about the foods they were eating (96.0\%), using appropriate size plates $(94.6 \%)$, not having a television that can be seen or heard during meals/snacks (93.1\%), making fruits and vegetables easier to eat (92.4\%) and having pleasant non-food conversations during meals/snacks (90.8\%; Table 1). The positive feeding practice least frequently observed (seen in less than 15\% of homes) included allowing children to serve themselves most/all food $(0.0 \%)$. The most common negative feeding practices included: enforcing table manners (88.6\%), rushing children to eat $(56.5 \%)$, when a child ate less than half of a meal or snack, the provider removed the plate without asking the child if s(he) was full (54.1\%) and ignoring or showing indifference to the children during the meal (49.6\%). The least frequent negative feeding practices included: eating unhealthy foods and beverages in front of 
Table 1 Frequency of new and existing food practices assessed using the modified Environment and Policy Assessment and Observation (EPAO) instrument in 133 family childcare homes $(\mathrm{FCCH})$ in Rhode Island and North Carolina, USA

\begin{tabular}{l}
\hline \% of FCCH where \\
Item
\end{tabular}

Positive feeding practices

The provider encouraged children to try $96 \cdot 2$ the foods on their plates

The provider talked with the children about the foods they were eating

During meals and snacks, did the provider use child size appropriate plates?*

Home does not have television that can be seen or heard from the eating area, or television in home but not on during meal

Did the provider make fruits and vegetables easier to eat?*

Lead/encourage pleasant non-food conversations during meals ${ }^{*}, \dagger$

The provider sat with the children

Did the provider encourage the children to sit around the table during meals?*

Did the provider take a moment with the children to settle before eating?*

Talk on the phone, text or work on the computer during meals* ${ }^{*} \dagger$

Provider ate the same foods as the children

When a child ate less than half of a meal or snack, the provider asked the child if (s)he was full before removing the plate

The provider praised a child for trying new or less preferred foods

Were children involved in meal preparation, planning or clean-up?*

Provider ate fruits or vegetables in front of children

Reason with a child to eat ${ }^{*}, \dagger$

Were a variety of healthy foods visible to children?*

Negotiate with a child to eat healthy foods*,$\dagger$

The provider enthusiastically role modelled eating healthy foods

The provider used an authoritative feeding style

Talk about feelings of hunger or fullness with children* ${ }^{*} \dagger$

Second helpings served only after a child requested seconds and the provider asked the child if (s)he was still hungry

Let a child choose between two healthy food options* ${ }^{*} \dagger$

Children served themselves most/all food and decided what portions to take

Negative feeding practices

Enforce table manners ${ }^{*}, \dagger$

Insist that a child eat a certain food ${ }^{*}, \dagger$

Spoon-feed a child to get them to eat ${ }^{\star}, \dagger$

Second helpings were served to a child, even when the child did not ask for more

Rush a child or children to eat ${ }^{\star}, \dagger$

When a child ate less than half of a meal or snack, the provider removed the plate without asking the child if $\mathrm{s}$ (he) was full

Table 1 Continued

\begin{tabular}{|c|c|}
\hline Item & $\begin{array}{l}\% \text { of } \mathrm{FCCH} \text { where } \\
\text { practice occurred }\end{array}$ \\
\hline $\begin{array}{l}\text { Ignore or show indifference to children } \\
\text { during the meal }{ }^{*}, \dagger\end{array}$ & $49 \cdot 6$ \\
\hline $\begin{array}{l}\text { Prompt a child to finish food already on } \\
\text { the plate in order to receive } \\
\text { seconds }{ }^{*}, \dagger\end{array}$ & $46 \cdot 6$ \\
\hline $\begin{array}{l}\text { Praise a child for cleaning his/her } \\
\text { plate }^{\star} \dagger\end{array}$ & $44 \cdot 3$ \\
\hline $\begin{array}{l}\text { Allow a child to have or take multiple } \\
\text { servings of a food, when more than } \\
\text { one food or a large amount of one } \\
\text { food remains on the plate* } \dagger\end{array}$ & 38.9 \\
\hline $\begin{array}{l}\text { The provider pressured a child to eat } \\
\text { more than they seemed to want }\end{array}$ & 38.4 \\
\hline $\begin{array}{l}\text { Were unhealthy snack foods visible to } \\
\text { children? }\end{array}$ & $38 \cdot 2$ \\
\hline $\begin{array}{l}\text { The provider used food as a reward or a } \\
\text { bribe for eating a less preferred food }\end{array}$ & 31.5 \\
\hline $\begin{array}{l}\text { Use food as reward for eating a specific } \\
\text { food }^{*}, \dagger\end{array}$ & $26 \cdot 7$ \\
\hline $\begin{array}{l}\text { The provider promised something other } \\
\text { than food for eating a specific food }\end{array}$ & 24.5 \\
\hline $\begin{array}{l}\text { Praise/compliment child for eating } \\
\text { unhealthy foods }{ }^{*}, \dagger\end{array}$ & $17 \cdot 6$ \\
\hline $\begin{array}{l}\text { Make special allowances to provide } \\
\text { something different from what was } \\
\text { already being served for a child who } \\
\text { refused to eat }{ }^{*}, \dagger\end{array}$ & $16 \cdot 7$ \\
\hline $\begin{array}{l}\text { The provider drank a soda or other } \\
\text { sweetened beverage in front of } \\
\text { children }\end{array}$ & $11 \cdot 3$ \\
\hline $\begin{array}{l}\text { When a child ate less than half of a } \\
\text { meal or snack, the provider required } \\
\text { the child sit at the table until (s)he } \\
\text { cleaned their plate }\end{array}$ & $10 \cdot 7$ \\
\hline $\begin{array}{l}\text { How often did the provider use food to } \\
\text { control a child's emotions?* }\end{array}$ & $10 \cdot 7$ \\
\hline $\begin{array}{l}\text { The provider used food as a reward or } \\
\text { withheld food as a punishment }\end{array}$ & $6 \cdot 3$ \\
\hline $\begin{array}{l}\text { The provider ate a salty snack in front of } \\
\text { children }\end{array}$ & 5.7 \\
\hline $\begin{array}{l}\text { The provider ate fast food in front of } \\
\text { children }\end{array}$ & 3.1 \\
\hline $\begin{array}{l}\text { The provider ate a sweet snack in front } \\
\text { of children }\end{array}$ & 1.9 \\
\hline
\end{tabular}

*Denotes new item (from additional twenty-five) on the EPAO Supplemental Assessment of Feding Practices.

†Lead in to these questions is 'How often did the provider...?'

children (i.e. sweet snacks (1.9\%), fast food (3.1\%), salty snacks $(5.7 \%)$ and sugary beverages (11.3\%)), using food as a reward/bribe $(6.3 \%)$ or to calm children's emotions (10.7\%) and requiring the child to sit at the table until the plate is clean $(10 \cdot 7 \%)$. 
indulgent feeding practices' and emerged with nineteen items and loadings ranging from 0.92 to 0.57 . Factor 2 appeared to capture 'autonomy support practices' and included ten items with factor loadings ranging from 0.68 to $0 \cdot 40$. It should be noted that one of the items had a low loading below the 0.40 cut-off (reason with children $=$ 0.397) but given its theoretical consistency with the factor, it was retained. Factor 3 appeared to capture "unhealthy role modelling' and included four items with loadings ranging from $0 \cdot 82$ to $0 \cdot 60$. The final three-factor solution captured $43 \%$ of total variance. Eigenvalues for the three factors were 13.84 for 'coercive control/indulgent practices', 3.94 for 'autonomy support practices' and 2.73 for 'unhealthy role modelling' (Table 2). Correlations among factors ranged from 0.20 to 0.46 (factor 1 and factor 2 , $r=0 \cdot 46$; factor 1 and factor 3, $r=0 \cdot 33$; factor 2 and factor 3 , $r=-0 \cdot 20$ ). Approximately half of the homes (50.4\%) had a 'high' autonomy supportive score that was above the median (0.59); $7 \cdot 0 \%$ had a 'high' unhealthy role modelling score based on the median split (1.0) and more than half (40.9\%) had a 'high' coercive controlling score based on the median split $(2 \cdot 7)$.

\section{Reliability (following exploratory factor analysis)}

Each of the three factors demonstrated good internal consistency (Cronbach's $\alpha>0 \cdot 70$ ). Values were as follows: 'coercive control/indulgent practices', $\alpha=0.97$; 'autonomy support practices', $\alpha=0.78$; and 'unhealthy role modelling', $\alpha=0.86$ (Table 2).

\section{Predictive validity of provider feeding practices on children's diet quality}

Children in this sample had an average diet quality, as demonstrated by a mean HEI-2010 score of $58 \cdot 8$ (SD 10.49). Correlations between HEI-2010 scores and each of the factors were as follows (factor 1 and HEI-2010, $r=0.065$; factor 2 and HEI-2010, $r=0.07$; factor 3 and HEI-2-10, $r=0 \cdot 29$ ). Multilevel mixed-effects models found some significant associations between provider feeding practices and children's diet quality. In the initial model (including all feeding practices), 'coercive control/indulgent practices' and 'unhealthy role modelling' were not significant. In addition, the covariates provider income, education, age, CACFP participation, quality rating and BMI, as well as the covariates child age and BMI, were not contributing significantly to the model. Hence, these were removed in the final reduced model. In the final model (Table 3), 'autonomy support practices' remained significantly associated with children's HEI-2010 scores. A 1unit increase in the use of 'autonomy support practices' was associated with a 9.4-unit increase in child HEI-2010 score. The covariates provider race, child sex, hours spent in child care and study arm, and the other two feeding constructs were also retained in the final model.

\section{Discussion}

Little is known about how childcare providers' feeding practices influence the diet and eating habits of young children in their care. This gap in knowledge is due in part to a lack of robust and comprehensive measurement tools, which we have tried to address in the present study using a sample of FCCH providers. Through our study, we identified feeding practices that were missing from current measurement tools, developed new items to capture these feeding practices and conducted psychometric testing with new items and scales. Results suggest that FCCH providers use a myriad of feeding practices while interacting with children during meal and snacks, and providers' use of autonomy-supportive practices is associated with higher diet quality in children. Future studies should continue to examine the use and impact of provider feeding practices, especially in different types of childcare settings (e.g. childcare centres, Head Start centres). Although our data are cross-sectional and the causal direction of the associations cannot be established, results suggest that interventions aimed at increasing providers' use of autonomysupportive practices may be a promising strategy for encouraging healthier eating habits in young children.

\section{Providers' use of feeding practices}

Our findings suggest that childcare providers use a variety of feeding practices, both positive and negative, which is consistent with previous literature in childcare. Similar to what providers report in qualitative studies, we observed that most childcare providers use positive feeding practices such as encouraging children to try new foods, providing nutrition education, creating a positive meal atmosphere and sitting with children during meals ${ }^{(49,50)}$. However, other positive feeding practices considered as best practice and promoted in childcare standards of practice $^{(10,51)}$ were observed less frequently, such as enthusiastically role modelling or assessing children's hunger before serving seconds.

There was also variation in the negative practices observed. For example, insisting children eat certain foods or spoon-feeding children appeared to be common practice. However, pressuring children to eat more food than they wanted, using food as a bribe to eat less preferred foods and modelling of unhealthy foods were not observed as frequently, which is consistent with past selfreported feeding practices of childcare providers $^{(14,25,37,52,53)}$. Overall, the findings from the present study offer further evidence that there continues to be room for improvement in the feeding practices being used in childcare settings ${ }^{(15,16)}$.

\section{Factors emerging from analysis}

The present study's EFA helps advance our understanding of how to conceptualize feeding practices in 
Table 2 Results of the exploratory factor analysis of the modified Environment and Policy Assessment and Observation (EPAO) instrument ${ }^{\star}, \dagger$,

\begin{tabular}{|c|c|c|c|}
\hline Item & Factor 1 & Factor 2 & Factor 3 \\
\hline \multicolumn{4}{|l|}{ Factor 1: 'Coercive control and indulgent feeding practices' $(a=0.97)$} \\
\hline The provider used food as a reward or withheld food as a punishment & 0.92 & 0.02 & 0.05 \\
\hline $\begin{array}{l}\text { When a child ate less than half of a meal or snack, the provider removed the plate } \\
\text { without asking the child if } s(\text { he) was full }\end{array}$ & 0.90 & 0.05 & $0 \cdot 11$ \\
\hline $\begin{array}{l}\text { When a child ate less than half of a meal or snack, the provider required the } \\
\text { child sit at the table until (s)he cleaned their plate }\end{array}$ & 0.89 & -0.04 & 0.02 \\
\hline The provider used food as a reward or a bribe for eating a less preferred food & 0.88 & 0.12 & 0.00 \\
\hline $\begin{array}{l}\text { Make special allowances to provide something different from what was already } \\
\text { being served for a child who refused to eat }\end{array}$ & 0.86 & $0 \cdot 19$ & -0.02 \\
\hline The provider promised something other than food for eating a specific food & 0.86 & 0.14 & 0.01 \\
\hline Praise/compliment child for eating unhealthy foods & 0.86 & 0.02 & 0.00 \\
\hline Use food as a reward for eating a specific food & 0.84 & 0.09 & 0.06 \\
\hline The provider pressured a child to eat more than they seemed to want & 0.84 & -0.07 & 0.07 \\
\hline Prompt a child to finish food already on the plate in order to receive seconds & 0.78 & 0.08 & -0.23 \\
\hline Second helpings were served to a child, even when the child did not ask for more & 0.74 & -0.08 & -0.06 \\
\hline $\begin{array}{l}\text { Allow a child to have or take multiple servings of a food, when more than one food or a } \\
\text { large amount of one food remains on the plate }\end{array}$ & 0.73 & 0.17 & -0.13 \\
\hline Insist that a child eat a certain food & 0.73 & 0.04 & -0.03 \\
\hline Talk on the phone, text or work on the computer during meals & 0.72 & 0.00 & -0.18 \\
\hline Praise a child for cleaning his/her plate & 0.70 & 0.17 & 0.07 \\
\hline Ignore or show indifference to children during the meal & 0.59 & -0.02 & 0.11 \\
\hline Rush a child or children to eat & 0.59 & 0.07 & 0.37 \\
\hline Enforce table manners & 0.58 & 0.08 & -0.24 \\
\hline Spoon-feed a child to get them to eat & 0.57 & 0.01 & 0.05 \\
\hline \multicolumn{4}{|l|}{ Factor 2: 'Autonomy support practices' $(a=0.78)$} \\
\hline The provider talked with the children about the foods they were eating & 0.08 & 0.68 & -0.01 \\
\hline The provider enthusiastically role modelled eating healthy foods & -0.03 & 0.61 & 0.04 \\
\hline The provider encouraged children to try the foods on their plates & 0.02 & 0.49 & 0.11 \\
\hline The provider praised a child for trying new or less preferred foods & -0.19 & 0.48 & 0.07 \\
\hline The provider used an authoritative feeding style & -0.04 & 0.47 & 0.07 \\
\hline Lead/encourage pleasant non-food conversations during meals & 0.30 & 0.46 & -0.05 \\
\hline Talk about feelings of hunger or fullness with children & 0.03 & 0.44 & -0.19 \\
\hline The provider sat with the children & $0 \cdot 18$ & 0.41 & 0.01 \\
\hline Did the provider make fruits and vegetables easier to eat? & 0.17 & 0.41 & -0.01 \\
\hline Reason with a child to eat & -0.19 & 0.40 & 0.13 \\
\hline Provider ate fruits or vegetables in front of children & 0.00 & 0.39 & 0.04 \\
\hline \multicolumn{4}{|l|}{ Factor 3: 'Negative role modelling' $(a=0.86)$} \\
\hline The provider ate a sweet snack in front of children & 0.05 & 0.21 & 0.82 \\
\hline The provider ate a salty snack in front of children & 0.07 & 0.08 & 0.81 \\
\hline The provider ate fast food in front of children & 0.17 & 0.12 & 0.80 \\
\hline The provider drank a soda or other sweetened beverage in front of children & 0.01 & $0 \cdot 13$ & 0.60 \\
\hline Eigenvalue & & 3.94 & 2.73 \\
\hline Variance (\%) & $28 \cdot 84$ & $8 \cdot 21$ & 5.69 \\
\hline Sum of variance captured (\%) & & $42 \cdot 75$ & \\
\hline
\end{tabular}

*Extraction method: maximum likelihood.

†Rotation method: oblimin with Kaiser normalization (oblique).

łLoadings above 0.40 are in bold.

Table 3 Reduced multivariable regression results examining the association between provider food practices and child Healthy Eating Index-2010 score in 133 family childcare homes in Rhode Island and North Carolina, USA

\begin{tabular}{lrrr}
\hline Final predictor & Estimate & $95 \% \mathrm{Cl}$ & $P$ value \\
\hline Factor 1 ('coercive control and indulgent feeding practices') & -5.3 & $-10.9,0.4$ & 0.07 \\
Factor 2 ('autonomy support practices') & 9.4 & $3.9,15.0$ & 0.001 \\
Factor 3 ('negative role modelling') & 5.8 & $-23.0,34.0$ & 0.69 \\
Provider race (other v. African American) & 4.2 & $0.13,8.2$ & 0.11 \\
Child sex (female v. male) & 2.3 & $0.5,4.1$ & 0.01 \\
Hours spent in childcare & 2.6 & $-0.8,5.9$ & 0.13 \\
Intervention $v$. control arm & 5.8 & $2.5,9.1$ & 0.001 \\
\hline
\end{tabular}

the childcare setting. Few previous childcare-based studies have used EFA to identify scales being measured by their instruments ${ }^{(54)}$. Results from our EFA suggested three factors: 'coercive control/indulgent practices', 'autonomy support practices' and 'unhealthy role modelling'. 
'Coercive control/indulgent practices' had the largest number of items as well as the highest eigenvalue and percentage of variance, although less than half of the providers had a high score. The practices included within this factor were consistent with both coercive controlling practices (e.g. using pressure, bribes and rushing child to eat) and with practices that are more permissive in nature (e.g. ignoring a child, talking on a cell phone or texting during the meal). Both types of practice may interfere with a provider being able to effectively engage with a child during meals and to help support her/his development of internal cues of satiety and hunger. The loading of items within what has been described as a higher-order factor is consistent with the recently developed content map for food parenting practices ${ }^{(6)}$ although instead of two higherorder constructs, we observed only one. It is possible that within the childcare setting, where a provider is interacting with multiple children at one time, the type of permissive practices used are somewhat different from the practices used by a parent in the home setting.

The second factor, which captured 'autonomy support practices', was consistent with another higher-order construct described in the content map. Items loading on this factor assessed talking with children about foods they are eating (informal nutrition education), encouraging and praising children for trying new foods and role modelling healthy eating. Approximately half of the providers had a high autonomy supportive score.

Interestingly, the third factor captured more a specific practice rather than a higher-order construct, specifically 'unhealthy role modelling' including foods such as fast food, sweets and salty snacks. Although these practices were not high in frequency and very few providers had a high score, they appear to be an important construct within this context.

Overall, the factor structure of provider feeding practices appears to be very consistent with that of the parenting literature in that coercive controlling practices come together into one factor as do autonomy-supportive practices ${ }^{(5,56)}$, although the exact terminology to name the factors may vary. A key difference is that the higher-order construct of structure did not emerge as a factor in our data. A few of the items intended to measure structure-related practices (e.g. enthusiastic role modelling of healthy foods, pleasant non-food conversations) loaded on the autonomy support factor instead. Other structure-related practices (e.g. having healthy foods visible (accessibility), letting child choose between healthy options (guided choices), talking/texting during meals (distractions during meals)) failed to load well on any factor. It is possible that because there is some inherent structure already in place in a childcare setting, these structure-related practices may not be as relevant as in the home setting. There were also several structure-related practices, such as monitoring and having rules and limits, that were not assessed because they were viewed as less relevant in the childcare environment.

\section{Influence of providers' feeding practices on children's diet quality}

In testing the predictive validity of the provider practices with child diet quality in FCCH, we found that 'autonomy support practices' was the only factor significantly associated with higher HEI-2010 score. Many of the practices captured by individual items have been shown to be associated with higher food acceptance or healthier food intakes; for example, enthusiastically role modelling, talking with the children about the foods they are eating, sitting with the children and using reasoning are associated with healthier foods, consistent with other studies conducted in childcare centres ${ }^{(19,23-25)}$. Our results advance the field by not only considering individual feeding practices but rather by examining how a group of practices/constructs can influence diet quality. Our findings are also consistent with the parenting literature in that supportive practices, such as parents' use of an authoritative style, may allow children to better regulate their internal cues of satiety and hunger and hence have healthier diet quality and weight status ${ }^{(22,55)}$. It is possible that in a childcare setting the use of autonomy-supportive practices is associated with diet quality while other practices are not, due to the setting. Unlike the home setting, children in childcare may respond more positively to certain practices while being less influenced by others. For example, in the home setting children may be less responsive to their primary caregiver's use of supportive practices given the less structured setting, while in childcare they may be expected to listen and respond given the group setting.

\section{Strengths, limitations and lessons learned}

The present study had many strengths including a thorough process to modify an existing tool that included expert review and two pilot studies, creation and use of videos to train data collectors, and assessment of provider feeding practices and child diet intake via direct observation. There are, however, some limitations. In our efforts to be parsimonious in adding items into an observation protocol, we may have not fully captured all possible practices that impact child diet quality in this setting. For example, some feeding practices were captured with a single item (e.g. offering encouragement to try new foods, involving the child in meal preparation, distractions like texting during meals). The brevity with which some practices were measured may have contributed EFA results that seemed to identify more of these overarching constructs rather than distinct practices. Use of items loading significantly on only one of the three factors might overlook some important practices. We found three items that did not load on any factors but were significantly correlated to HEI-2010 score (i.e. encourage children to sit at the table, healthy foods are visible to children in the home, use of appropriate child size plates). Another 
challenge when measuring feeding practices was the need to standardize our scoring to account to variation in length of the childcare day and number of meals and snacks eaten at childcare. Our weighting of feeding practices based on time spent in eating occasions was an attempt to create this standardization, but alternative methods could be considered such as weighting based on eating occasion as a meal $v$. snack. Another limitation of the study is its reliance on the larger ongoing intervention trial for our sample and data collection. These FCCH are but a sample of $\mathrm{FCCH}$ in the area ${ }^{(58)}$. FCCH willing to participate in an intervention study may represent a unique subset of $\mathrm{FCCH}$, which may limit generalizability. In addition, it is possible that the intervention homes may have changed their feeding practices from baseline to follow-up; however, we controlled for this in our analysis. Future studies with larger samples should continue to explore how items that capture more environmental aspects might fit into other constructs. Finally, our results may not be generalizable to other childcare settings; FCCH are a specific type of childcare whereby a provider takes care of children in his or her home and may differ from a childcare centre, where the number of children and the policies that are in place may differ and have an impact on mealtime interactions.

\section{Conclusions}

Similar to the parenting literature, constructs which describe coercive controlling practices and those that describe autonomy-supportive practices emerged. We found that childcare providers' use of autonomysupportive practices in FCCH was associated with a higher HEI-2010 score. Similar to the parenting literature there is a continued need for efforts that focus training and education on positive practices rather than just eliminating negative ones such pressure and rewards ${ }^{(58)}$. Given that diets of pre-schoolers in the USA continue to be suboptimal, teaching childcare providers about what they can be doing instead of what they should not be doing may help improve the children's diet quality.

\section{Acknowledgements}

Acknowledgements: The authors would like to thank Megan Fallon for her assistance with coding the videos and for her contribution to the literature review. They would also like all participants who contributed to this study. Financial support: This work was supported by the National Institutes of Health, Bethesda, MD, USA (grant number R01HL108390) and early career diversity grant funds (A.T., grant number 3R01HL108390-03S1). The funders had no role in the design, analysis or writing of this article. Conflict of interest: None of the authors have any conflict of interest to disclose. Authorship: All authors contributed to the various stages of this study. A.T. contributed to the study design, performed some of the statistical analyses and drafted the manuscript. A.E.V. and S.E.B.N. contributed to the study design and helped draft the manuscript. T.O. and J.O.F. participated in discussion of the study design and revised the manuscript. K.W. conducted the exploratory factor analysis and helped revise the manuscript. T.L. conducted the multilevel analysis and helped revise the manuscript. R.B. helped manage data collection and read the manuscript. D.S.W. conceived of the initial idea for the study, contributed to the study design, revised the manuscript and contributed especially to the intellectual content. All the authors read and commented on the drafts and approved the final version for submission. Ethics of human subject participation: This study was conducted according to the guidelines laid down in the Declaration of Helsinki and all procedures involving human subjects were approved by the Institutional Review Board at the University of Rhode Island (first pilot study) and the Institutional Review Boards at the University of North Carolina at Chapel Hill, Duke University and the University of Rhode Island (second pilot study). Written informed consent was obtained from all subjects.

\section{References}

1. Skinner JD, Carruth BR, Wendy B et al. (2002) Children's food preferences: a longitudinal analysis. J Am Diet Assoc 102, 1638-1647.

2. Cashdan E (1994) A sensitive period for learning about food. Hum Nat 5, 279-291.

3. Dwyer JT, Suitor CW \& Hendricks K (2004) FITS: new insights and lessons learned. J Am Diet Assoc 104, 1 Suppl. 1, S5-S7.

4. Davison KK \& Birch LL (2001) Childhood overweight: a contextual model and recommendations for future research. Obes Rev 2, 159-171.

5. Ritchie LD, Welk G, Styne D et al. (2005) Family environment and pediatric overweight: what is a parent to do? $\mathrm{J} \mathrm{Am}$ Diet Assoc 105, 5 Suppl. 1, S70-S79.

6. Vaughn AE, Ward DS, Fisher JO et al. (2016) Fundamental constructs in food parenting practices: a content map to guide future research. Nutr Rev 74, 98-117.

7. US Census Bureau, Public Information Office (2013) Child Care Costs on the Upswing. Census Bureau Reports 2013. https:// www.census.gov/newsroom/press-releases/2013/cb13-62. html (accessed November 2017).

8. Mamedova S \& Redford J (2013) Early Childhood Program Participation, From the National Household Education Surveys Program of 2012: First Look. Washington, DC: National Center for Education Statistics, Institute of Education Sciences, US Department of Education.

9. American Dietetic Association (2005) Position of the American Dietetic Association: benchmarks for nutrition programs in child care settings. J Am Diet Assoc 105, 979-986.

10. Benjamin Neelon SE \& Briley ME; American Dietetic Association (2011) Position of the American Dietetic Association: benchmarks for nutrition in child care. J Am Diet Assoc 111, 607-615. 
11. US Department of Agriculture \& US Department of Health and Human Services (2015) 2015-2020 Dietary Guidelines for Americans, 8th ed. Washington, DC: US Government Printing Office.

12. Nicklas TA, Baranowski T, Baranowski JC et al. (2001) Family and child-care provider influences on preschool children's fruit, juice, and vegetable consumption. Nutr Rev 59, 224-235.

13. Story M, Kaphingst KM \& French S (2006) The role of child care settings in obesity prevention. Future Child 16, 143-168.

14. Ramsay SA, Branen LJ, Fletcher J et al. (2010) 'Are you done?' Child care providers' verbal communication at mealtimes that reinforce or hinder children's internal cues of hunger and satiation. J Nutr Educ Behav 42, 265-270.

15. Dev DA, McBride BA, Speirs KE et al. (2014) Predictors of Head Start and child-care providers' healthful and controlling feeding practices with children aged 2 to 5 years. J Acad Nutr Diet 114, 1396-1403.

16. Martyniuk OJ, Vanderloo LM, Irwin JD et al. (2016) Comparing the nutrition environment and practices of home- and centrebased child-care facilities. Public Health Nutr 19, 575-584.

17. Ahn R \& Nelson MR (2015) Observations of food consumption in a daycare setting. Young Consum 16, 420-437.

18. Elford L \& Brown A (2014) Exploring child-feeding style in childcare settings: how might nursery practitioners affect child eating style and weight? Eat Behav 15, 314-317.

19. Kharofa RY, Kalkwarf HJ, Khoury JC et al. (2016) Are mealtime best practice guidelines for child care centers associated with energy, vegetable, and fruit intake? Child Obes 12, 52-58.

20. Hendy HM (1999) Comparison of five teacher actions to encourage children's new food acceptance. Ann Behav Med 21, 20-26.

21. Hendy HM \& Raudenbush B (2000) Effectiveness of teacher modeling to encourage food acceptance in preschool children. Appetite 34, 61-76.

22. Ward S, Blanger M, Donovan D et al. (2017) Association between childcare educators' practices and preschoolers' physical activity and dietary intake: a cross-sectional analysis. BMJ Open 7, e013657.

23. Gubbels JS, Gerards SM \& Kremers SP (2015) Use of food practices by childcare staff and the association with dietary intake of children at childcare. Nutrients 7, 2161-2175.

24. Gubbels JS, Kremers SP, Stafleu A et al. (2010) Child-care environment and dietary intake of 2- and 3-year-old children. J Hum Nutr Diet 23, 97-101.

25. Anundson K, Sisson SB, Anderson M et al. (2017) Staff foodrelated behaviors and children's tastes of food groups during lunch at child care in Oklahoma. J Acad Nutr Diet $\mathbf{1 1 8}$, 1399-1407.

26. Vereecken C, Huybrechts I, Maes L et al. (2008) Food consumption among preschoolers. Does the school make a difference? Appetite 51, 723-726.

27. Patrick H, Hennessy E, McSpadden K et al. (2013) Parenting styles and practices in children's obesogenic behaviors: scientific gaps and future research directions. Child Obes $\mathbf{9}$, Suppl., S73-S86.

28. Dev DA, Carraway-Stage V, Schober DJ et al. (2017) Implementing the Academy of Nutrition and Dietetics benchmarks for nutrition education for children: child-care providers' perspectives. J Acad Nutr Diet 117, 1963-1971. e2.

29. Dev DA \& McBride BA; STRONG Kids Research Team (2013) Academy of Nutrition and Dietetics benchmarks for nutrition in child care 2011: are child-care providers across contexts meeting recommendations? J Acad Nutr Diet $\mathbf{1 1 3}$, $1346-1353$

30. Freedman MR \& Alvarez KP (2010) Early childhood feeding: assessing knowledge, attitude, and practices of multi-ethnic child-care providers. J Am Diet Assoc 110, 447-451.
31. Ward DS, Mazzucca S, McWilliams C et al. (2015) Use of the Environment and Policy Evaluation and Observation as a Self-Report Instrument (EPAO-SR) to measure nutrition and physical activity environments in child care settings: validity and reliability evidence. Int J Behav Nutr Phys Act 12, 124.

32. Ward D, Hales D, Haverly K et al. (2008) An instrument to assess the obesogenic environment of child care centers. Am J Health Behav 32, 380-386.

33. Ostbye T, Mann CM, Vaughn AE et al. (2015) The keys to healthy family child care homes intervention: study design and rationale. Contemp Clin Trials 40, 81-89.

34. Vaughn AE, Mazzucca S, Burney R et al. (2017) Assessment of nutrition and physical activity environments in family child care homes: modification and psychometric testing of the Environment and Policy Assessment and Observation. BMC Public Health 17, 680.

35. Ward D, Morris E, McWilliams C et al. (2014) Go NAP SACC: Nutrition and Physical Activity Self-Assessment for Child Care, 2nd ed. Chapel Hill, NC: Center for Health Promotion and Disease Prevention and Department of Nutrition, University of North Carolina at Chapel Hill; available at https:// gonapsacc.org/resources/nap-sacc-materials

36. Ward DS, Vaughn AE, Mazzucca S et al. (2017) Translating a child care based intervention for online delivery: development and randomized pilot study of Go NAPSACC. BMC Public Health 17, 891.

37. Fallon M, Halloran K, Gorman K et al. (2018) Self-reported and observed feeding practices of Rhode Island Head Start teachers: knowing what not to do. Appetite 120, 310-317.

38. Tovar A, Vaughn AE, Fallon M et al. (2016) Providers' response to child eating behaviors: a direct observation study. Appetite 105, 534-541.

39. Hughes SO, Patrick H, Power TG et al. (2007) The impact of child care providers' feeding on children's food consumption. J Dev Behav Pediatr 28, 100-107.

40. Ball SC, Benjamin SE \& Ward DS (2007) Development and reliability of an observation method to assess food intake of young children in child care. J Am Diet Assoc 107, 656-661.

41. Guenther PM, Casavale KO, Reedy J et al. (2013) Update of the Healthy Eating Index: HEI-2010. J Acad Nutr Diet 113, 569-580.

42. US Department of Agriculture \& US Department of Health and Human Services (2010) Dietary Guidelines for Americans, 2010, 7th ed. Washington, DC: US Government Printing Office.

43. Guenther PM, Kirkpatrick SI, Reedy J et al. (2014) The Healthy Eating Index-2010 is a valid and reliable measure of diet quality according to the 2010 Dietary Guidelines for Americans. J Nutr 144, 399-407.

44. Erinosho TO, Ball SC, Hanson PP et al. (2013) Assessing foods offered to children at child-care centers using the Healthy Eating Index-2005. J Acad Nutr Diet 113, 1084-1089.

45. Hanson KL \& Olson CM (2013) School meals participation and weekday dietary quality were associated after controlling for weekend eating among US school children aged 6 to 17 years. J Nutr 143, 714-721.

46. Romo-Palafox MJ, Ranjit N, Sweitzer SJ et al. (2018) Contribution of beverage selection to the dietary quality of the packed lunches eaten by preschool-aged children. $J$ Acad Nutr Diet 118, 1417-1424.

47. Ziegler A (2011) Generalized Estimating Equations. New York: Springer.

48. Morrissey TW \& Banghart P (2007) Family Child Care in the United States. http://www.nccp.org/publications/pub_720. html (accessed November 2017).

49. Lindsay AC, Salkeld JA, Greaney ML et al. (2015) Latino family childcare providers' beliefs, attitudes, and practices related to promotion of healthy behaviors among preschool children: a qualitative study. J Obes 2015, 409742.

50. Vandeweghe L, Moens E, Braet C et al. (2016) Perceived effective and feasible strategies to promote healthy eating in 
young children: focus groups with parents, family child care providers and daycare assistants. BMC Public Health 16, 1045.

51. American Academy of Pediatrics, American Public Health Association \& National Resource Center for Health and Safety in Child Care (2010) Preventing Childhood Obesity in Early Care and Education: Selected Nutrition and Physical Activity Standards. Aurora, CO: National Resource Center for Health and Safety in Child Care and Early Education.

52. Elford L \& Brown A (2014) Exploring child-feeding style in childcare settings: how might nursery practitioners affect child eating style and weight? Eat Behav 15, 314-317.

53. Maalouf J, Evers SC, Griffin M et al. (2013) Assessment of mealtime environments and nutrition practices in child care centers in Georgia. Child Obes 9, 437-445.

54. Gubbels JS, Sleddens EF, Raaijmakers L et al. (2016) The Child-care Food and Activity Practices Questionnaire
(CFAPQ): development and first validation steps. Public Health Nutr 19, 1964-1975.

55. Yee AZ, Lwin MO \& Ho SS (2017) The influence of parental practices on child promotive and preventive food consumption behaviors: a systematic review and meta-analysis. Int J Behav Nutr Phys Act 14, 47.

56. Shloim N, Edelson LR, Martin N et al. (2015) Parenting styles, feeding styles, feeding practices, and weight status in 4-12 year-old children: a systematic review of the literature. Front Psychol 6, 1849.

57. Ward DS, Vaughn AE, Burney RV et al. (2016) Recruitment of family child care homes for an obesity prevention intervention study. Contemp Clin Trials Commun 3, 131-138.

58. Johnson SL (2016) Developmental and environmental influences on young children's vegetable preferences and consumption. Adv Nutr 7, issue 1, 220S-231S. 